\title{
Synthesis of nanographenes, starphenes and sterically congested polyarenes by aryne cyclotrimerization
}

Iago Pozo, Enrique Guitián, Dolores Pérez and Diego Peña*

Centro Singular de Investigación en Química Biolóxica e Materiais Moleculares (CiQUS) and Departamento de Química Orgánica, Universidade de Santiago de Compostela. 15782 Santiago de Compostela (Spain).

Email corresponding author: diego.pena@usc.es 

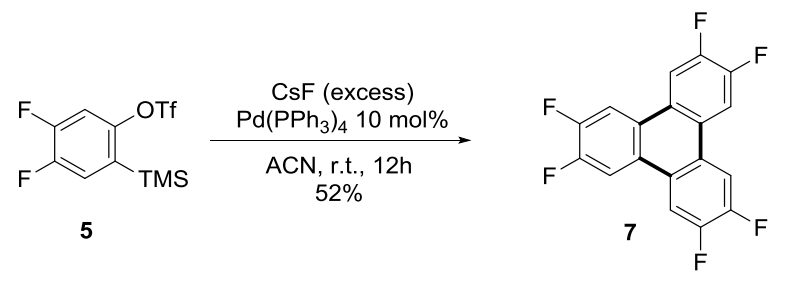

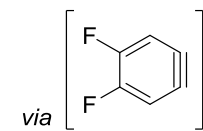

6

Scheme S1. ${ }^{1}$

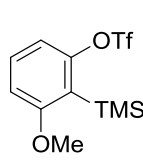

8

CsF (excess) $\underset{\mathrm{ACN} \text {, r.t., } 12 \mathrm{~h}}{\stackrel{\mathrm{Pd}\left(\mathrm{PPh}_{3}\right)_{4} 10 \mathrm{~mol} \%}{8}}$ $81 \%$<smiles>Cc1ccc(C)c(S(C)(=O)=O)c1[O+]</smiles>

12

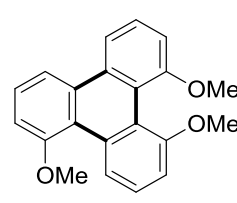

10<smiles>COc1cccc2c1c1cccc(OC)c1c1cccc(OC)c21</smiles>

11 via $[\underbrace{}_{\text {OMe }}$

9

Scheme S2. ${ }^{1}$

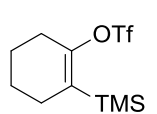

15

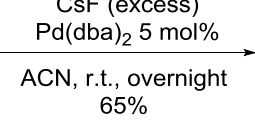

$65 \%$

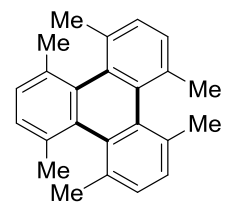

14

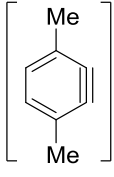

13

\section{Scheme S3. ${ }^{2}$}

$$
\begin{gathered}
\mathrm{CsF} \text { (excess) } \\
\stackrel{\mathrm{Pd}\left(\mathrm{PPh}_{3}\right)_{4} 10 \mathrm{~mol} \%}{\mathrm{ACN}, \text { r.t., } 12 \mathrm{~h}} \\
64 \%
\end{gathered}
$$

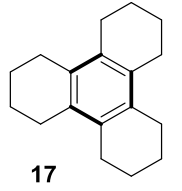

17

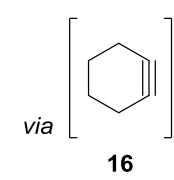

Scheme $54{ }^{3}$ 

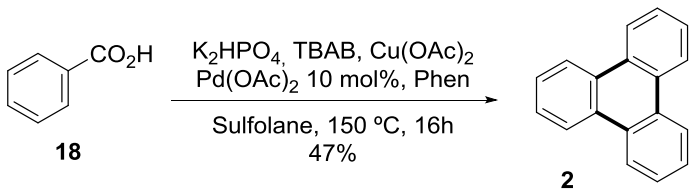

via

1

Scheme $55 .{ }^{4}$
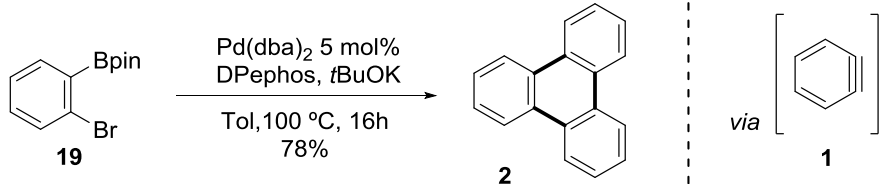

Scheme $56{ }^{5}$

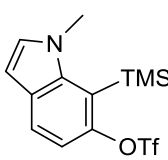

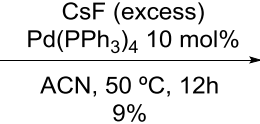
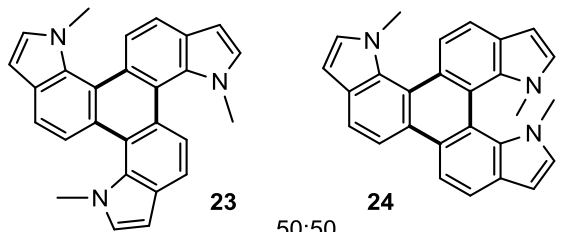

50:50

Scheme $57{ }^{6}$

OTf

CsF (excess) $\mathrm{Pd}(\mathrm{dba})_{2} 10 \mathrm{~mol} \%$

BINAP

ACN $, 50^{\circ} \mathrm{C}, 12$ $93 \%$
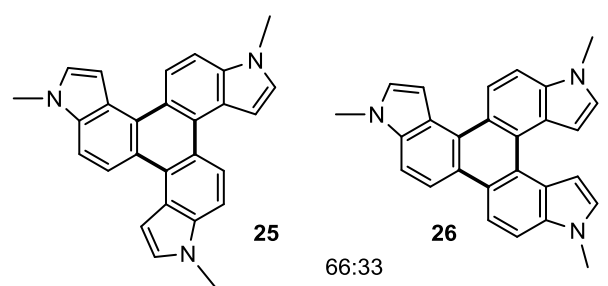

via<smiles>Cn1ccc2ccccc21</smiles>

21

Scheme S8. ${ }^{6}$ 


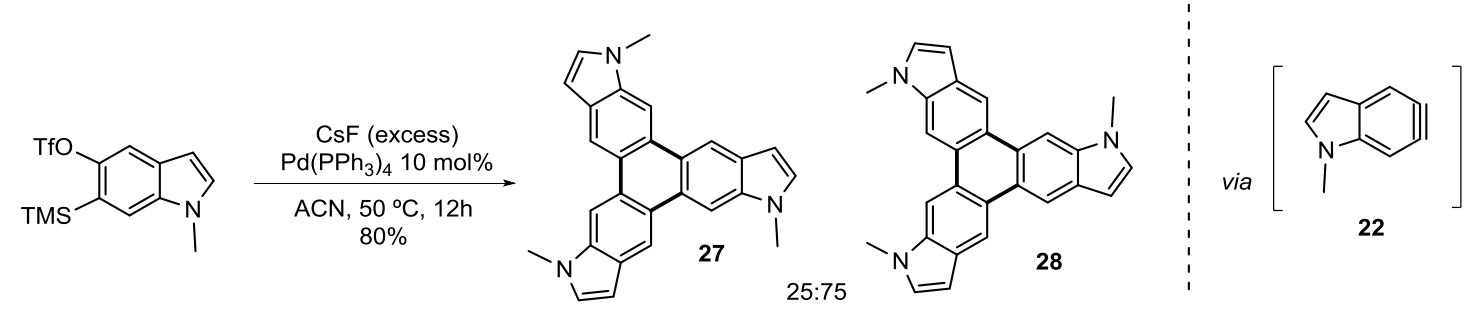

Scheme S9. ${ }^{6}$

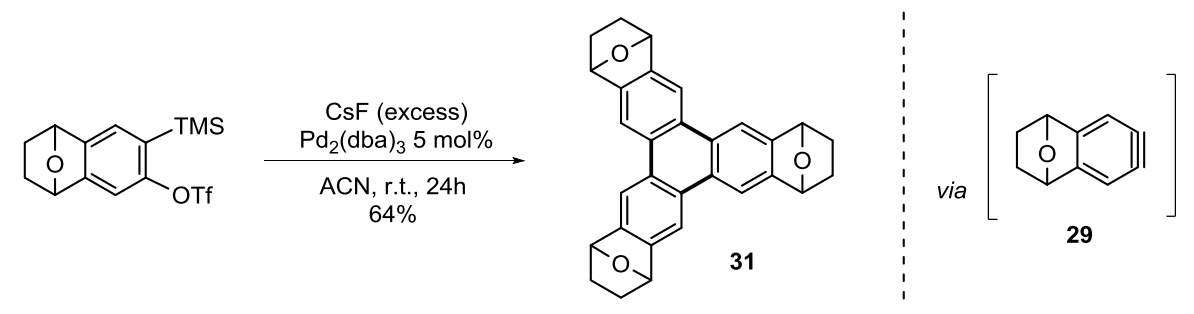

Scheme S10. ${ }^{7}$

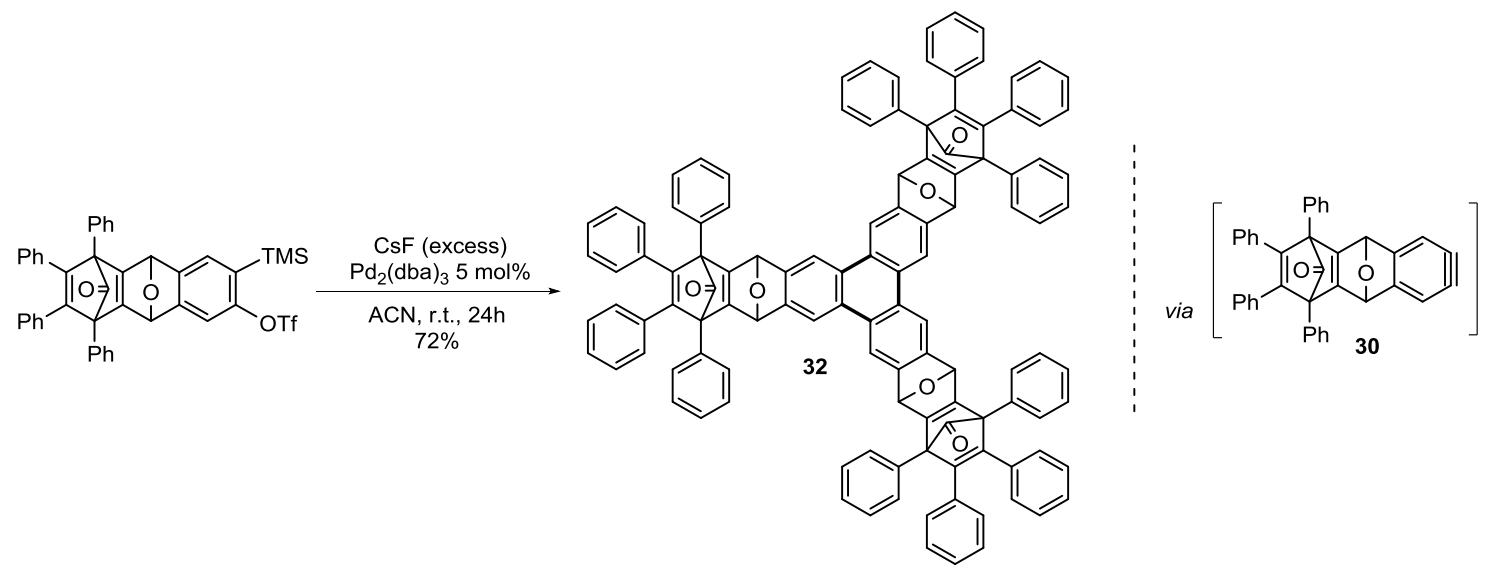

Scheme S11. ${ }^{7}$
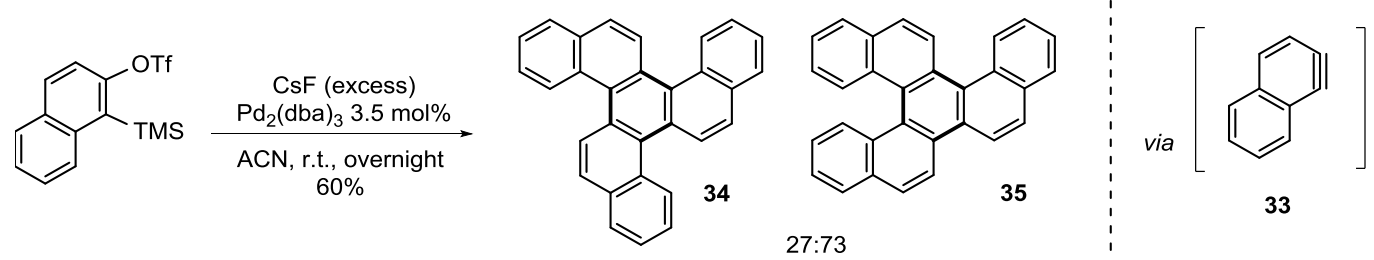

33

Scheme S12. ${ }^{8}$ 


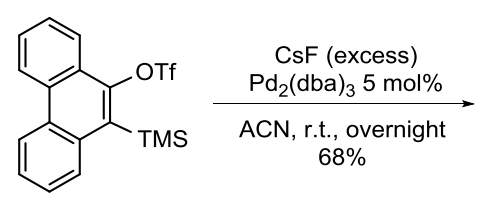

64

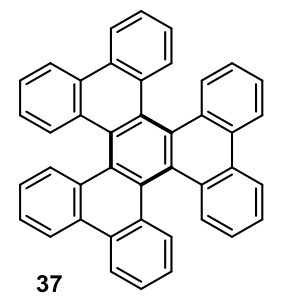

37

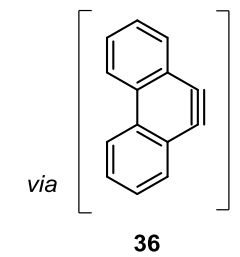

36

Scheme S13. ${ }^{9}$
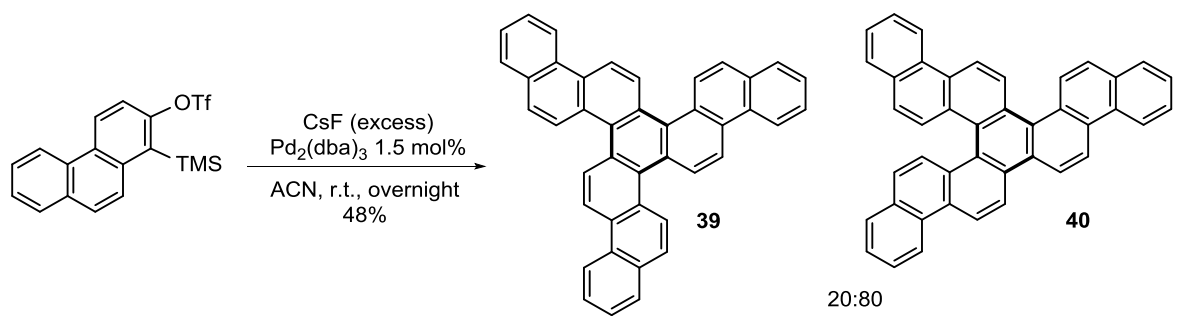

Scheme S14. ${ }^{10}$
TMS \begin{tabular}{c}
$\mathrm{CsF}$ (excess) \\
$\mathrm{Pd}_{2}(\mathrm{dba})_{3} 5 \mathrm{~mol} \%$ \\
\hline $\mathrm{ACN}$, r.t., $12 \mathrm{~h}$ \\
$26 \%$
\end{tabular}
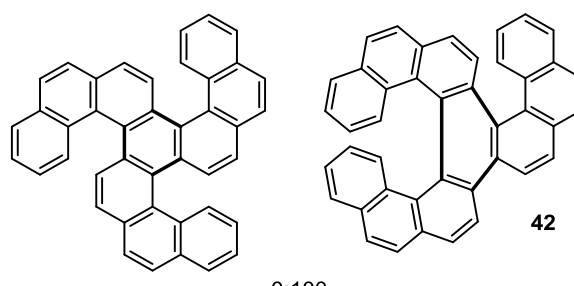

$0: 100$

Scheme S15. ${ }^{11}$
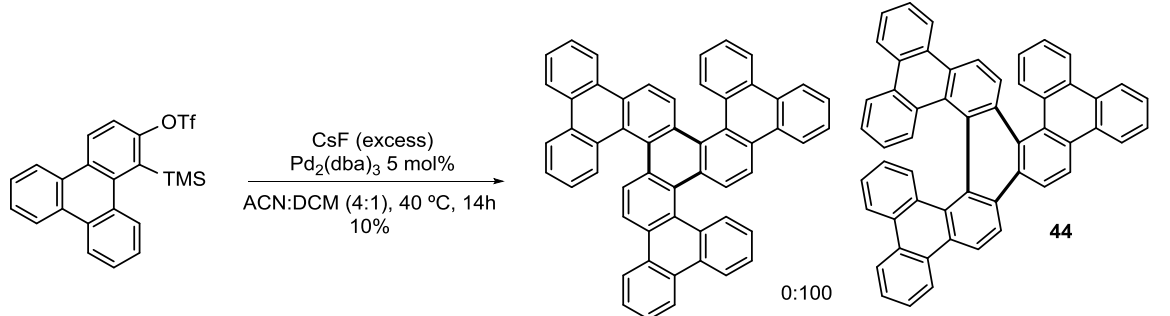

via

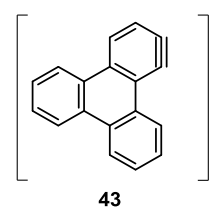

Scheme S16. ${ }^{12}$ 
<smiles></smiles>

CsF (excess) $\mathrm{Pd}_{2}(\mathrm{dba})_{3} 10 \mathrm{~mol} \%$ $\mathrm{ACN}$, r.t., 2
$54-56 \%$

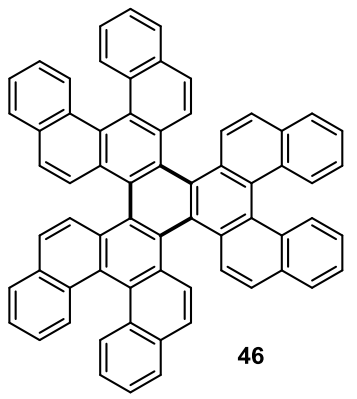

Scheme S17. ${ }^{13-14}$

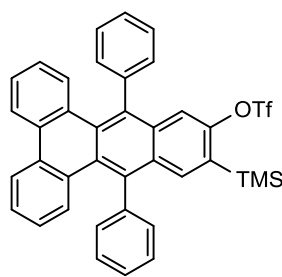

CsF (excess) $\mathrm{Pd}\left(\mathrm{PPh}_{3}\right)_{4} 10 \mathrm{~mol} \%$ $22 \%$
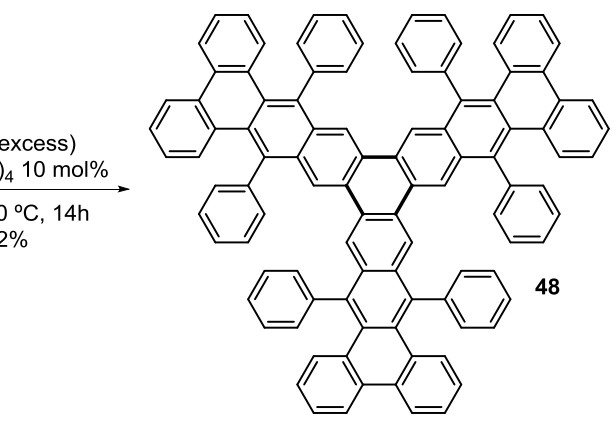

Scheme S18. ${ }^{15}$
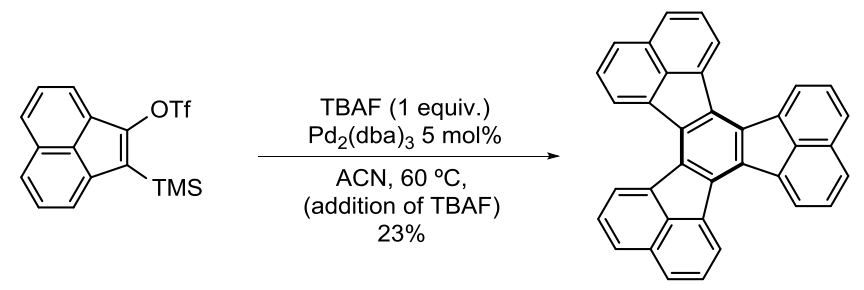

50

Scheme S19. ${ }^{3}$

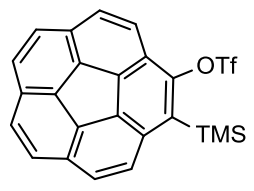

CsF (excess) $\mathrm{Pd}_{2}(\mathrm{dba})_{3} 10 \mathrm{~mol} \%$ ACN:DCM
$(4: 1)$, r.t., overnight $40 \%$
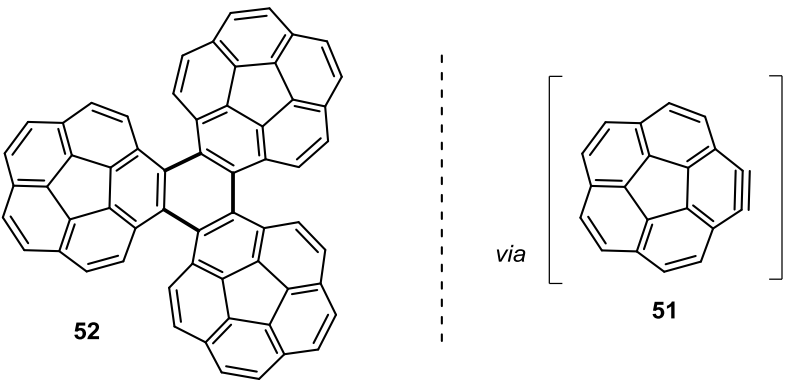

Scheme S20. ${ }^{16}$ 


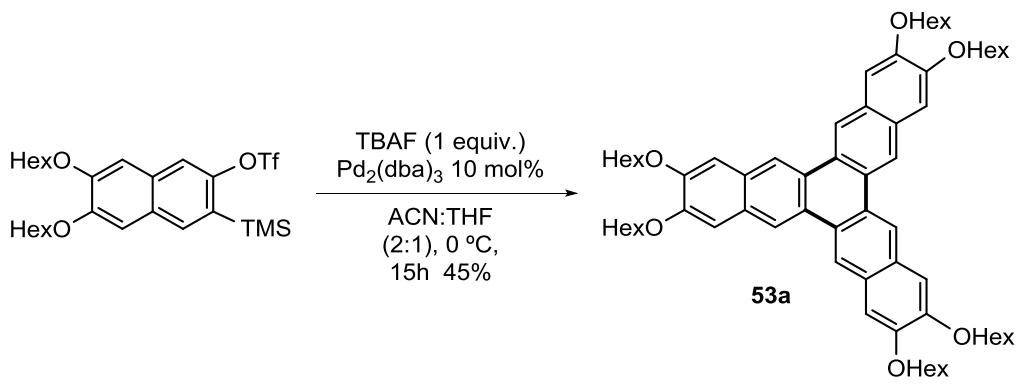

Scheme S21. ${ }^{17}$

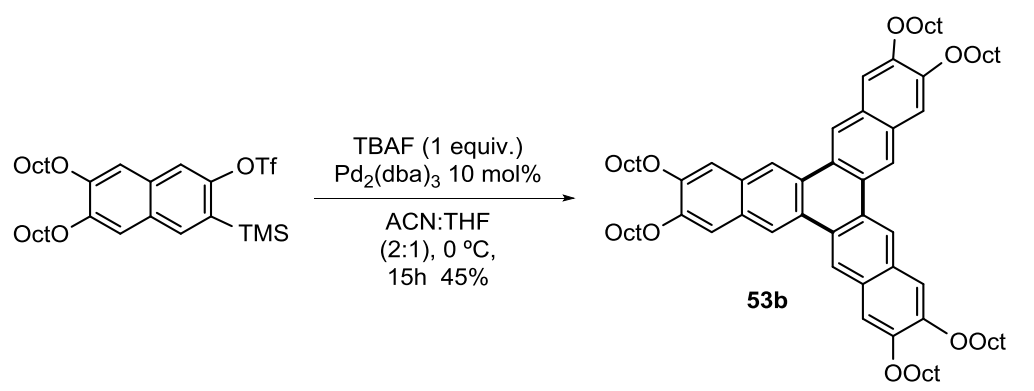

Scheme S22. ${ }^{17}$
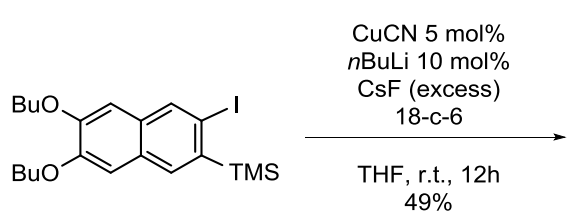

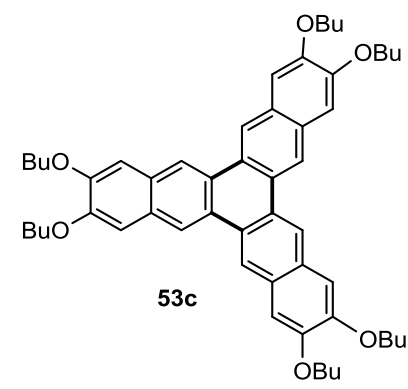

Scheme $523 .^{18}$

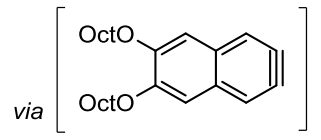

54b via<smiles>CCCCOc1cc2cc#ccc2cc1OCCC</smiles>

54c

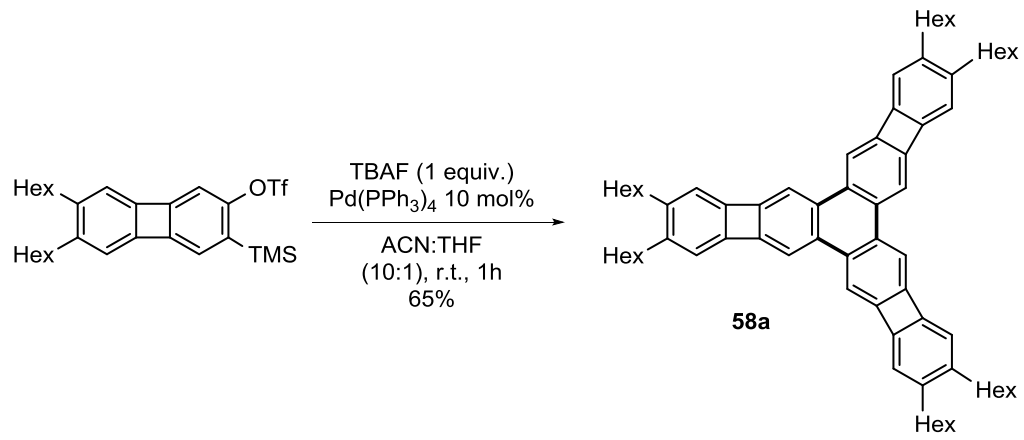

Scheme S24. ${ }^{19}$

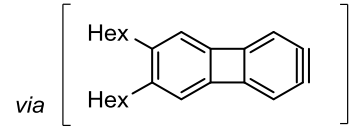

$57 a$ 


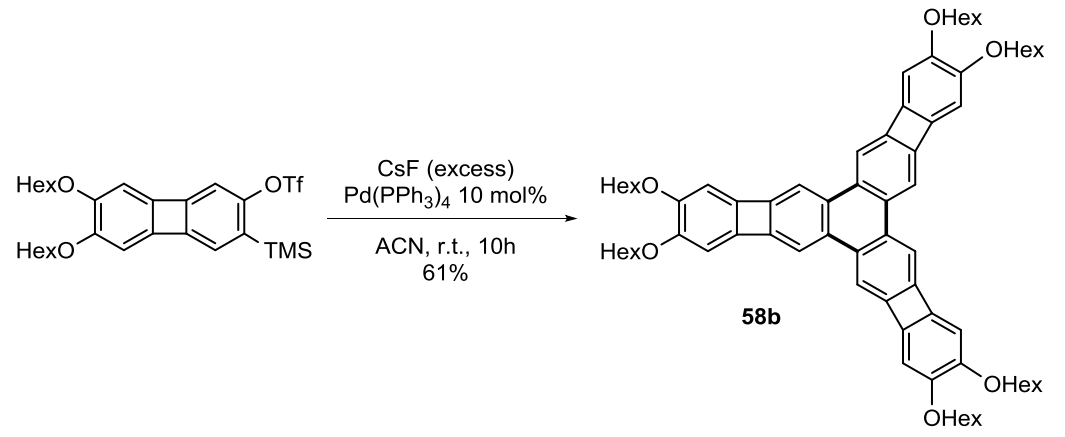

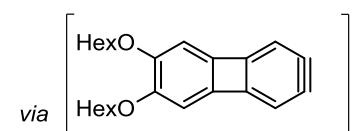

$57 b$

Scheme S25. ${ }^{20}$

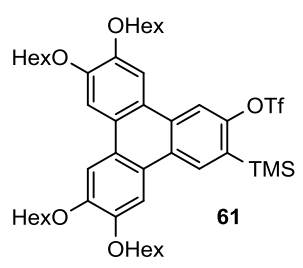

CsF (excess) $\mathrm{Pd}\left(\mathrm{PPh}_{3}\right)_{4} 10 \mathrm{~mol} \%$

ACN:DCM
$(3: 1)$, r.t., $14 \mathrm{~h}$ $28 \%$
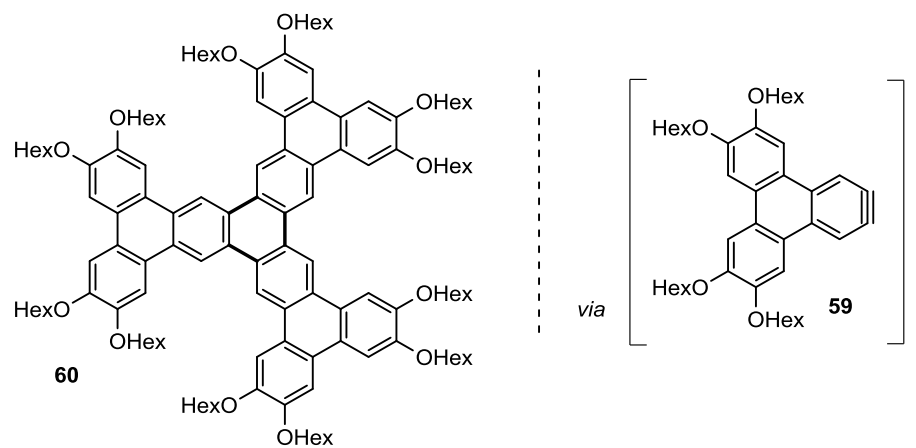

Scheme S26. ${ }^{21}$

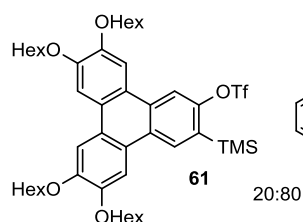

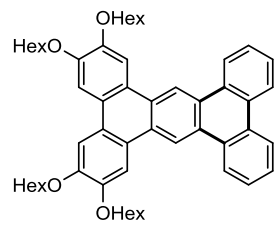

62

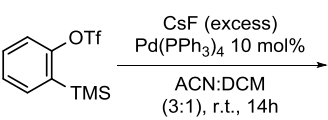

Hex

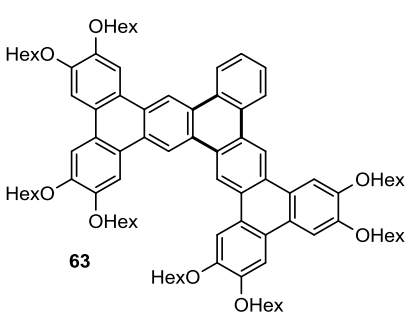

Scheme $527 .^{21}$

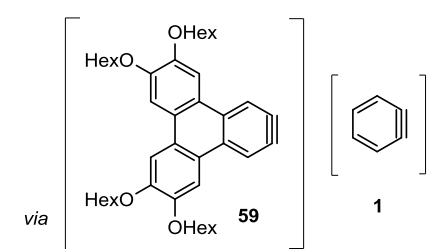



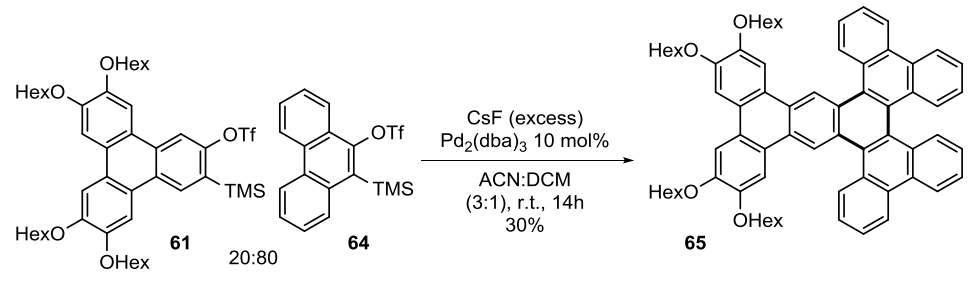

Scheme $528 .{ }^{22}$
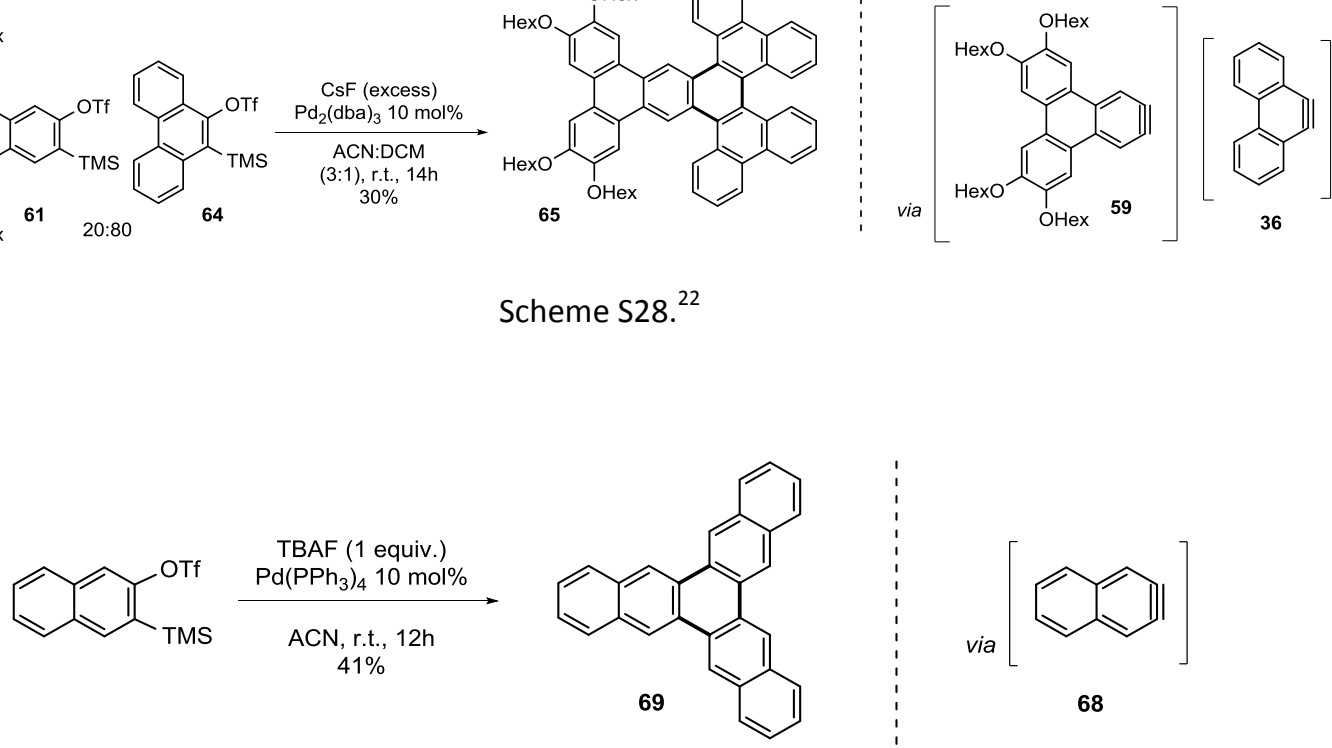

36

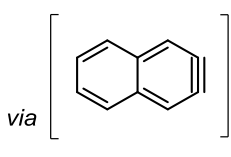

68

Scheme S29. ${ }^{23}$
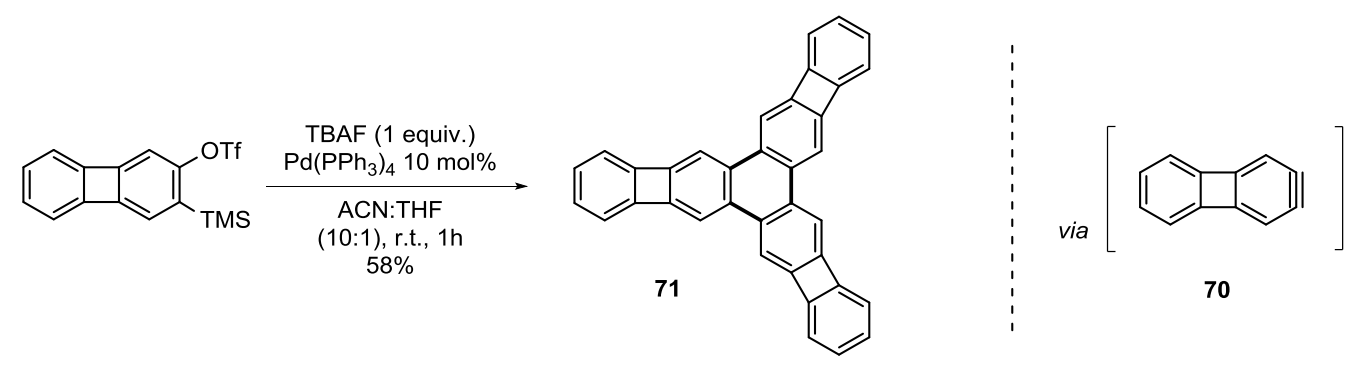

Scheme S30. ${ }^{19}$
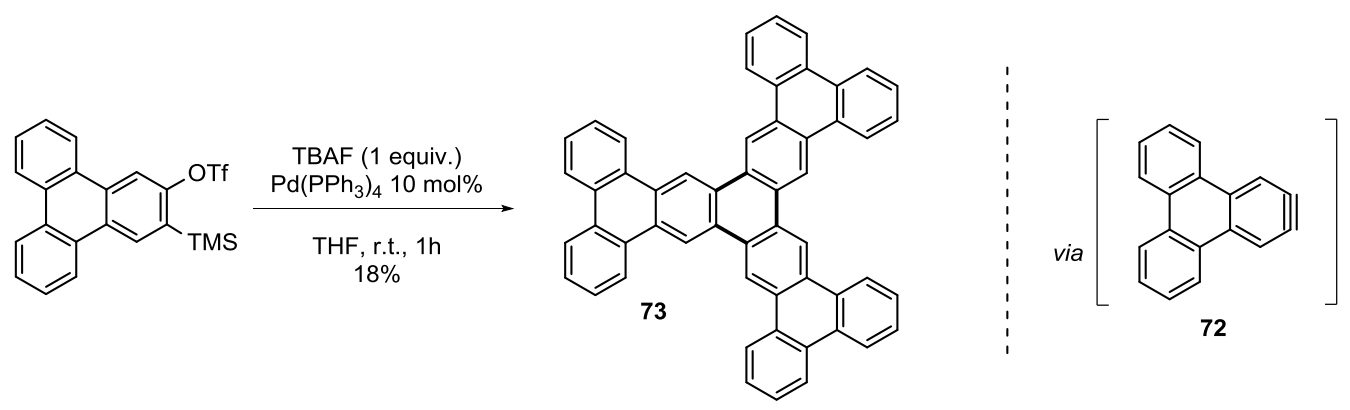

Scheme S31. ${ }^{21}$ 


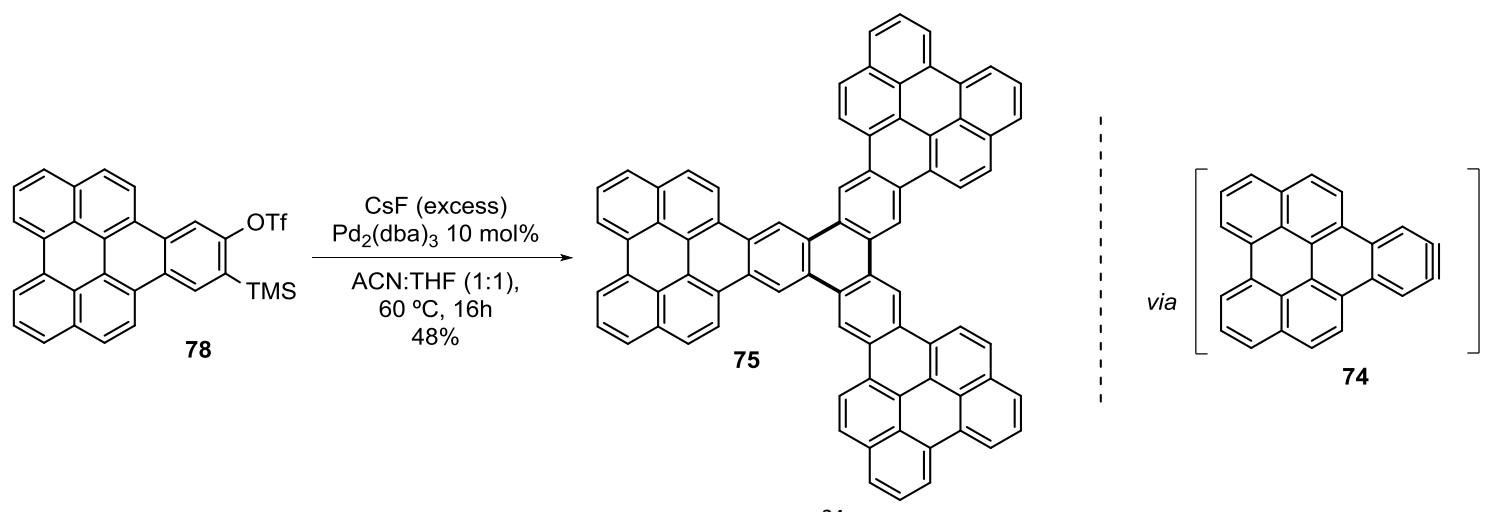

Scheme S32. ${ }^{24}$
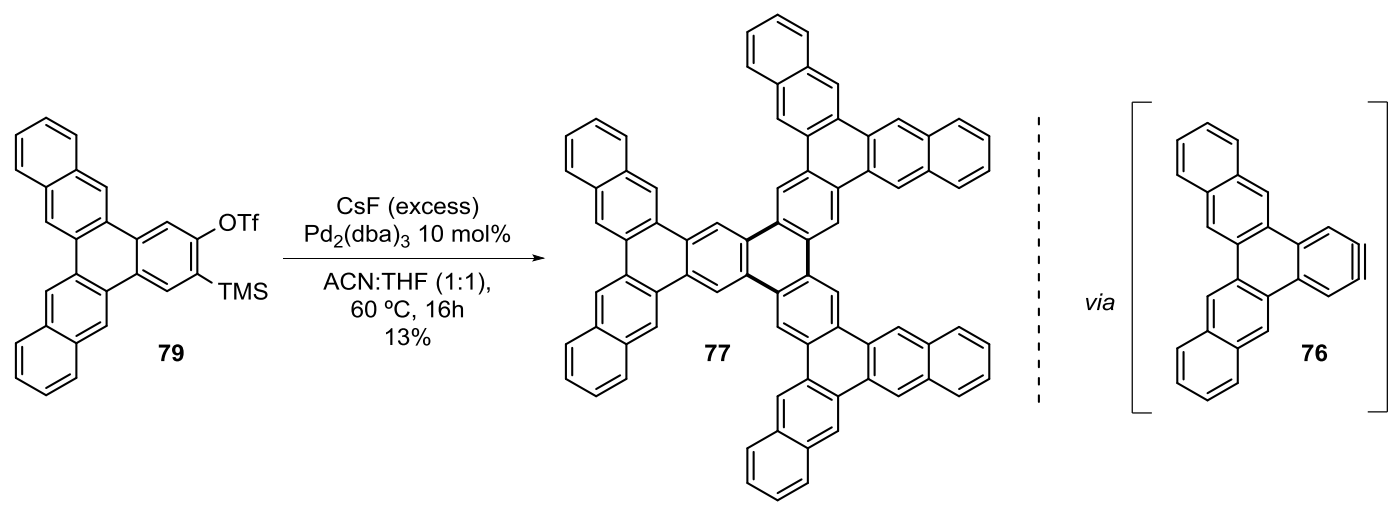

Scheme S33. ${ }^{25}$

S10 


\section{References}

1. Peña, D.; Escudero, S.; Pérez, D.; Guitián, E.; Castedo, L. Efficient Palladium-Catalyzed Cyclotrimerization of Arynes: Synthesis of Triphenylenes Angew. Chem. Int. Ed. 1998, 37, 2659-2661.

2. Wang, Y.; Stretton, A. D.; McConnell, M. C.; Wood, P. A.; Parsons, S.; Henry, J. B.; Mount, A. R.; Galow, T. H. 1,4,5,8,9,12-Hexamethyltriphenylene. A Molecule with a Flipping Twist J. Am. Chem. Soc. 2007, 129, 13193-13200.

3. Iglesias, B.; Peña, D.; Pérez, S.; Guitián, E.; Castedo, L. Palladium-catalyzed Trimerization of Strained Cycloalkynes: Synthesis of Decacyclene Synlett 2002, 3, 486-488.

4. Cant, A. A.; Roberts, L.; Greaney, M. F. Generation of benzyne from benzoic acid using $\mathrm{C}-\mathrm{H}$ activation Chem. Commun. 2010, 46, 8671-8673.

5. Garcia-Lopez, J.; Greaney, M. F. Use of 2-Bromophenylboronic Esters as Benzyne Precursors in the Pd- Catalyzed Synthesis of Triphenylenes Org. Lett. 2014, 16, 2338.2341.

6. Lin, J. B.; Shah, T. K.; Goetz, A. E.; Garg, N. K.; Houk, K. N. Conjugated Trimeric Scaffolds Accessible from Indolyne Cyclotrimerizations: Synthesis, Structures, and Electronic Properties J. Am. Chem. Soc. 2017, 139, 10447-10455.

7. Tozawa, H.; Kakuda, T.; Adachi, K.; Hamura, T. Star-Shaped Polycyclic Aromatic Ketones via 3-Fold Cycloadditions of Isobenzofuran Trimer Equivalent Org. Lett. 2017, 19, 4118-4121.

8. Peña, D.; Pérez, D.; Guitián, E.; Castedo, L. Synthesis of Hexabenzotriphenylene and Other Strained Polycyclic Aromatic Hydrocarbons by Palladium-Catalyzed Cyclotrimerization of Arynes Org. Lett. 1999, $1,1555-1557$.

9. Peña, D.; Cobas, A.; Pérez, D.; Guitián, E.; Castedo, L. Kinetic Control in the Palladium-Catalyzed Synthesis of C2-Symmetric Hexabenzotriphenylene. A Conformational Study Org. Lett. 2000, 2, 16291632.

10. Palladium-Catalized Cycloaddition Reactions of Arynes. Top. Organomet. Chem. 2005, 14: 109-146.

11. Peña, D.; Cobas, A.; Pérez, D.; Guitián, E.; Castedo, L. Dibenzo[a,o]phenanthro[3,4-s]pycene, a Configurationally Stable Double Helicene: Synthesis and Determination of Its Conformation by NMR and GIAO Calculations Org. Lett. 2003, 5, 1863-1866.

12. Romero, C.; Peña, D.; Pérez, S.; Guitián, E. Palladium-Catalyzed [2+2+2] Cycloadditions of 3,4Didehydrophenanthrene and 1,2-Didehydrotriphenylene J. Org. Chem. 2008, 73, 7996-8000.

13. Hosokawa, T.; Takahashi, Y.; Matsushima, T.; Watanabe, S.; Kikkawa, S.; Azumaya, I.; Tsurusaki, A.; Kamikawa, K. Synthesis, Structures, and Properties of Hexapole Helicenes: Assembling Six [5]Helicene Substructures into Highly Twisted Aromatic Systems J. Am. Chem. Soc. 2017, 139, 1851218521.

14. Zuzak, R.; Castro-Esteban, J.; Brandimarte, P.; Engelund, M.; Cobas, A.; Pi.tkowski, P.; Kolmer, M.; Perez, D.; Guitian, E.; Szymonski, M.; Sanchez-Portal, D.; Godlewski, S.; Pena, D. Building a 22-ring nanographene by combining in-solution and on-surface syntheses Chem. Commun. 2018, 54, 1025610259.

15. Alonso, J. M.; Díaz-Álvarez, A. E.; Criado, A.; Pérez, D.; Peña, D.; Guitián, E. [16]Cloverphene: a Clover-Shaped cata-Condensed Nanographene with Sixteen Fused Benzene Rings Angew. Chem. Int. Ed. 2012, 51, 173-177. 
16. Yanney, M.; Fronczek, F. R.; Henry, W. P.; Beard, D. J.; Sygula, A. Cyclotrimerization of Corannulyne: Steric Hindrance Tunes the Inversion Barriers of Corannulene Bowls Eur. J. Org. Chem. 2011, 6636-6639.

17. Lynett, P. T.; Maly, K. E. Synthesis of Substituted Trinaphthylenes via Aryne Cyclotrimerization Org. Lett. 2009, 11, 3726-3729.

18. Hein, S. J.; Lehnherr, D.; Dichtel, W. R. Rapid access to substituted 2-naphthyne intermediates via the benzannulation of halogenated silylalkynes Chem. Sci. 2017, 8, 5675-5681.

19. Iglesias, B.; Cobas, A.; Pérez, D.; Guitián, E. Tris(benzocyclobutadieno)triphenylene and Its Lower Biphenylene Homologues by Palladium-Catalyzed Cyclizations of 2,3-Didehydrobiphenylene Org. Lett. 2004, 6, 3557-3560.

20. Godlewski, S.; Engelund, M.; Peña, D.; Zuzak, R.; Kawai, H.; Kolmer, M.; Caeiro, J.; Guitián, E.; Vollhardt, K. P. C.; Sánchez-Portal, D.; Szymonski, M.; Pérez, D. Site-selective reversible Diels-Alder reaction between a biphenylene-based polyarene and a semiconductor surface Phys. Chem. Chem. Phys. 2018, 20, 11037-11046.

21. Romero, C.; Peña, D.; Pérez, S.; Guitián, E. Synthesis of Extended Triphenylenes by PalladiumCatalyzed [2+2+2] Cycloaddition of Triphenylynes Chem. Eur. J. 2006, 12, 5677-5684.

22. Rodríguez-Lojo, D.; Peña, D.; Pérez, S.; Guitián, E. Aryne-mediated syntheses of structurally related acene derivatives Org. Biomol. Chem. 2010, 8, 3386-3388.

23. Soe, W.; Manzano, C.; Renaud, N.; Mendoza, P.; Sarkar A. D., Ample, F.; Hliwa, M.; Echavarren, A. M.; Chandrasekhar, N.; Joachim C. Manipulating Molecular Quantum States with Classical Metal Atom Inputs: Demonstration of a Single Molecule NOR Logic Gate ACS Nano 2011, 5, 1436-1440.

24. Schuler, B.; Collazos, S.; Gross, L.; Meyer, G.; Pérez, D.; Guitián, E.; Peña, D. From Perylene to a 22-Ring Aromatic Hydrocarbon in One-Pot Angew. Chem. Int. Ed. 2014, 53, 9004-9006.

25. Vilas-Varela, M.; Fatayer, S.; Majzik, Z.; Pérez, D.; Guitián, E.; Gross, L.; Peña, D. [19]Dendriphene: A 19-Ring Dendritic Nanographene Chem. Eur. J. 2018, 24, 17697-17700. 